PROCEEDINGS OF THE

AMERICAN MATHEMATICAL SOCIETY

Volume 33, Number 2, June 1972

\title{
SOME WALLMAN COMPACTIFICATIONS DETERMINED BY RETRACTS
}

\author{
H. L. BENTLEY
}

\begin{abstract}
Let $Y$ be a Hausdorff compactification of a locally compact space $X$ and let $K=Y-X$ be the remainder. Suppose that $K$ is a regular Wallman space (i.e. $K$ has a base for closed sets which is a ring and which consists of sets each of which is the closure of its interior) and suppose that $K$ is a neighoorhood retract of $Y$. Under these assumptions, it is proved below that $Y$ is a Wallman compactification of $X$.
\end{abstract}

I. Introduction. Throughout this paper all topological spaces are assumed to be Hausdorff. We shall rely on the references [1]-[4], [6], [9]-[12] for background material and basic definitions and notations. The ideas are due to Frink [6] who considered a normal base $\mathscr{F}$ of closed subsets of a space $X$ and defined a compactification $w(\mathscr{F})$ called a Wallman compactification. It is still an open question whether every compactification $Y$ of a Tychonoff space $X$ is of the form $w(\mathscr{F})$ for a suitable choice of $\mathscr{F}$. We provide some partial results in the case when $X$ is locally compact.

II. Normal bases on locally compact spaces. Throughout this paper, $X$ denotes a fixed locally compact space, $Y$ denotes a fixed compactification of $X$, and $K$ denotes the remainder $Y-X$. Thus, $K$ is compact. We now recall a few results from the author's paper [2]. $L$ is co-compact in $X$ iff $\mathrm{Cl}_{X}(X-L)$ is compact. If $\mathscr{H}$ ' is any family of closed subsets of $X$, then the compact modification of the family $\mathscr{H}$ denoted $\mathrm{CM}(\mathscr{H})$, is the family of subsets of $X$ of the form $(H \cap L) \cup B$ where $H \in \mathscr{H}, L$ is co-compact in $X$, and $B$ is a compact subset of $X$.

It is proved in [2] that if $\mathscr{F}$ is a normal base on $X$ such that $\varnothing, X \in \mathscr{F}$, then $\operatorname{CM}(\mathscr{F})$ is also a normal base and $w(\mathscr{F})=w(\operatorname{CM}(\mathscr{F}))$. On the other hand, $\mathrm{CM}(\mathscr{H})$ may be a normal base even though $\mathscr{H}$ is not and herein lies the usefulness of compact modifications.

Presented to the Society, January 22, 1971; received by the editors April 28, 1971. AMS 1970 subject classifications. Primary 54D35, 54D45, 54C15; Secondary 54C20.

Key words and phrases. Wallman compactification, normal base, locally compact space, retract.

(c) American Mathematical Society 1972 
THEOREM 1. Let $\mathscr{H}$ be a ring of closed subsets of $X$ such that $\varnothing, X \in \mathscr{H}$. Let $\mathscr{F}=\mathrm{CM}(\mathscr{H})$ and let $\mathscr{F}=\left\{\mathrm{Cl}_{Y}(F) \mid F \in \mathscr{F}\right\}$. Then $\mathscr{F}$ has the trace property with respect to $X$ iff whenever $H_{1}, \cdots, H_{n} \in \mathscr{H}$ such that $K \cap \bigcap_{i=1}^{n} \mathrm{Cl}_{Y}\left(H_{i}\right) \neq \varnothing$, and whenever $L$ is co-compact in $X$, we have $L \cap \bigcap_{i=1}^{n} H_{i} \neq \varnothing$.

The proof is straightforward and is omitted.

THEOREM 2. Let $\mathscr{H}$ be a ring of closed subsets of $X$ such that $\varnothing, X \in \mathscr{H}$. Let $\mathscr{F}=\mathrm{CM}(\mathscr{H})$, let $\mathscr{F}=\left\{\mathrm{Cl}_{Y}(F) \mid F \in \mathscr{F}\right\}$, and suppose $\mathscr{F}$ is separating

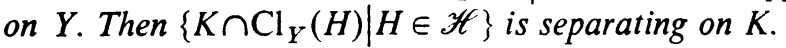

The proof is not difficult and is omitted. The following lemma presents a partial converse of Theorem 2. Its proof, while somewhat intricate, is primarily an application of the algebra of sets; at any rate, the proof is a straightforward generalization of the proof of the main theorem of the author's paper [2]. Thus, no proof is supplied here.

Lemma 3. Let $\mathscr{H}$ be a ring of closed subsets of $X$ such that $\varnothing, X \in \mathscr{H}$ and suppose that $\mathscr{H}$ also satisfies the condition: If $H \in \mathscr{H}$ such that $K \subset \mathrm{Cl}_{Y}(H)$, then $H$ is co-compact in $X$. Let $\mathscr{F}=\mathrm{CM}(\mathscr{H})$ and let $\mathscr{F}=$ $\left\{\mathrm{Cl}_{Y}(F) \mid F \in \mathscr{F}\right\}$. Let $\mathscr{G}=\left\{K \cap \mathrm{Cl}_{Y}(H) \mid H \in \mathscr{H}\right\}$ and suppose that $\mathscr{G}$ is a separating ring on $K$. Then $\mathscr{F}$ is separating on $Y$.

III. Retracts. We now consider the consequences of $K$ being a retract of $Y$ (recall the notational convention regarding $X, Y$, and $K$ established in $\S \mathrm{II})$. The following result is basic.

THEOREM 4. Let $f: Y \rightarrow K$ be a retract map. Let $L$ be co-compact in $X$. Then $f[L]$ is dense in $K$.

Proof. $K \subset \mathrm{Cl}_{Y}(L)$ so $K=f(K) \subset f\left(\mathrm{Cl}_{Y}(L)\right) \subset \mathrm{Cl}_{K}(f(L))$.

LeMMA 5. Let $f: Y \rightarrow K$ be a retract map and let $P$ be a regular closed subset of $K$. Then $P=K \cap \mathrm{Cl}_{Y}\left(X \cap f^{-1}(P)\right)$.

Proof. Since $X$ is dense in $Y$,

Thus,

$$
\mathrm{Cl}_{Y}\left(f^{-1}\left(\operatorname{Int}_{Y}(P)\right) \cap X\right)=\mathrm{Cl}_{Y}\left(f^{-1}\left(\operatorname{Int}_{Y}(P)\right)\right) \text {. }
$$

$$
\begin{aligned}
P & =\mathrm{Cl}_{K}\left[f^{-1}\left(\operatorname{Int}_{Y}(P)\right) \cap K\right] \subset \mathrm{Cl}_{Y}\left[f^{-1}\left(\operatorname{Int}_{Y}(P)\right)\right] \cap K \\
& =\mathrm{Cl}_{Y}\left[f^{-1}\left(\operatorname{Int}_{Y}(P)\right) \cap X\right] \cap K \\
& \subset \mathrm{Cl}_{Y}\left[f^{-1}(P) \cap X\right] \cap K \subset f^{-1}(P) \cap K=P .
\end{aligned}
$$

THEOREM 6. Let $f: Y \rightarrow K$ be a retract map and suppose $K$ is a regular Wallman space. Then $Y$ is a Wallman compactification of $X$. 
Proof. Let $\mathscr{G}^{\prime}$ be a separating ring of regular closed subsets of $K$. Let $\mathscr{G}=\mathscr{G}^{\prime} \cup\{\varnothing, K\}$. Then $\mathscr{G}$ is also a separating ring of regular closed subsets of $K$. Let $\mathscr{H}=\left\{X \cap f^{-1}(G) \mid G \in \mathscr{G}\right\}$. Let $\mathscr{F}=\mathrm{CM}(\mathscr{H})$ and let $\mathscr{F}=\left\{\mathrm{Cl}_{Y}(F) \mid F \in \mathscr{F}\right\}$.

In order to show $\mathscr{F}$ is separating on $Y$, we apply Lemma 3 . Let $H \in \mathscr{H}$ such that $K \subset \mathrm{Cl}_{Y}(H)$. It suffices to show that $H$ is co-compact in $X$. There is a $G \in \mathscr{G}$ such that $H=X \cap f^{-1}(G) . K=K \cap \mathrm{Cl}_{Y^{\prime}}(H)=$ $K \cap \mathrm{Cl}_{Y}\left(X \cap f^{-1}(G)\right)=G$ by Lemma 5 . Thus, $H=X$ and $H$ is co-compact in $X$.

To show that $\mathscr{F}$ has the trace property with respect to $X$, we use Theorem 1. Let $H_{1}, \cdots, H_{n} \in \mathscr{H}$ such that $K \cap \bigcap_{i=1}^{n} \mathrm{Cl}_{Y}\left(H_{i}\right) \neq \varnothing$ and let $L$ be co-compact in $X$. There exist $G_{1}, \cdots, G_{n} \in \mathscr{G}$ such that $H_{i}=$ $X \cap f^{-1}\left(G_{i}\right), i=1, \cdots, n$. Let $y \in K \cap \bigcap_{i=1}^{n} \mathrm{Cl}_{Y}\left(H_{i}\right)$. Then

$$
y \in \bigcap_{i=1}^{n} K \cap \mathrm{Cl}_{Y}\left(X \cap f^{-1}\left(G_{i}\right)\right)=\bigcap_{i=1}^{n} G_{i} \in \mathscr{G} .
$$

Let $G=\bigcap_{i=1}^{n} G_{i}$. Then $y \in G=\mathrm{Cl}_{K}\left(\operatorname{Int}_{K}(G)\right)$ so $\operatorname{Int}_{K}(G)$ is a nonempty open subset of $K$. Since $f[L]$ is dense in $K, f[L] \cap \operatorname{Int}_{K}(G) \neq \varnothing$. Thus, $f[L] \cap G \neq \varnothing$ which implies

$$
L \cap \bigcap_{i=1}^{n} H_{i}=L \cap \bigcap_{i=1}^{n} X \cap f^{-1}\left(G_{i}\right)=L \cap f^{-1}(G) \neq \varnothing .
$$

LEMMA 7. Let $Y_{1}$ and $Y_{2}$ be compactifications of $X$ such that $Y_{1} \geqq Y_{2}$. Suppose $Y_{1}-X$ is a retract of $Y_{1}$. Then $Y_{2}-X$ is a retract of $Y_{2}$.

Proof. Let $g: Y_{1} \rightarrow Y_{2}$ be the canonical quotient map. Let $K_{1}=Y_{1}-X$, let $K_{2}=Y_{2}-X$, and note that $g\left[K_{1}\right]=K_{2}$. Let $f: Y_{1} \rightarrow K_{1}$ be a retract map. Define the function $h: Y_{2} \rightarrow K_{2}$ by $h(x)=g(f(x))$ for $x \in X$ and $h(x)=x$ for $x \in K_{2}$. If $H$ is closed in $K_{2}$, then $h^{-1}(H)=g\left[f^{-1}\left(g^{-1}(H)\right)\right]$. Therefore, $h$ is continuous.

THEOREM 8. Let $Y_{1}$ and $Y_{2}$ be compactifications of $X$ such that $Y_{1} \geqq Y_{2}$. Let $K_{1}=Y_{1}-X$ and let $K_{2}=Y_{2}-X$. Suppose $K_{1}$ is a retract of $Y_{1}$ and suppose $K_{2}$ is a regular Wallman space. Then $Y_{2}$ is a Wallman compactification of $X$.

Proof. This theorem follows immediately from Lemma 7 and Theorem 6.

We turn now to a slightly different approach and assume that the $f$ image of every co-compact subset of $X$ is the same. We are able to get somewhat improved results in this case. We shall say that $S$ is the cocompact image of a continuous map $f: Y \rightarrow K$ if $S \subset K$ and also, whenever $L$ is a co-compact subset of $X$, we have $f[L]=S$. 
LEMMA 9. If $f: Y \rightarrow X$ is a retract map with a co-compact image $S$, then $S$ is dense in $K$ and $\mathrm{Cl}_{K}(P)=K \cap \mathrm{Cl}_{Y}\left(X \cap f^{-1}(P)\right)$ for each $P \subset S$.

Proof. Since $f^{-1}\left[\mathrm{Cl}_{K}(P)\right]$ is closed in $Y$,

Thus,

$$
\mathrm{Cl}_{Y}\left[f^{-1}(P) \cap X\right] \subset f^{-1}\left[\mathrm{Cl}_{K}(P)\right]
$$

$$
K \cap \mathrm{Cl}_{Y}\left[f^{-1}(P) \cap X\right] \subset K \cap f^{-1}\left[\mathrm{Cl}_{K}(P)\right]=\mathrm{Cl}_{K}(P) .
$$

Let $t \in P$. Since $f^{-1}(t)=\left[f^{-1}(t) \cap X\right] \cup\{t\}$ is compact, either $f^{-1}(t) \cap X$ is compact or $t \in \mathrm{Cl}_{Y}\left[f^{-1}(t) \cap X\right]$. The first alternative implies that $f\left[X-f^{-1}(t)\right]=S$, which is absurd in view of the fact that $t \in S$. Thus, $t \in K \cap \mathrm{Cl}_{Y}\left[f^{-1}(t) \cap X\right] \subset K \cap \mathrm{Cl}_{Y}\left[X \cap f^{-1}(P)\right]$ for each $t \in P$. Therefore, $\mathrm{Cl}_{K}(P) \subset K \cap \mathrm{Cl}_{Y}\left[X \cap f^{-1}(P)\right]$.

THEOREM 10. Let $f: Y \rightarrow K$ be a retract map with a co-compact image $S$ and assume $K$ is a Wallman compactification of $S$. Then $Y$ is a Wallman compactification of $X$.

Proof. Let $\mathscr{G}$ be a family of closed subsets of $S$ such that $\varnothing, S \in \mathscr{G}$. Let $\mathscr{H}=\left\{X \cap f^{-1}(G) \mid G \in \mathscr{G}\right\}$ and let $\mathscr{F}=\mathrm{CM}(\mathscr{H})$. Let

$$
\overline{\mathscr{G}}=\left\{\mathrm{Cl}_{K^{K}}(G) \mid G \in \mathscr{G}\right\}
$$

and let $\bar{F}=\left\{\mathrm{Cl}_{Y}(F) \mid F \in \mathscr{F}\right\}$. We shall actually prove slightly more than is required. Namely, we shall prove the following three statements:

(1) $\mathscr{F}$ has the trace property with respect to $X$ iff $\overline{\mathscr{G}}$ has the trace property with respect to $S$.

(2) $\overline{\mathscr{F}}$ is separating on $Y$ iff $\overline{\mathscr{G}}$ is separating on $K$.

(3) $Y \cong w(\mathscr{F})$ iff $K \cong w(\mathscr{G})$.

Proof of 1. Suppose $\mathscr{F}$ has the trace property with respect to $X$. Let $G_{1}, \cdots, G_{n} \in \mathscr{G}$ such that $\bigcap_{i=1}^{n} \mathrm{Cl}_{K^{-}}\left(G_{i}\right) \neq \varnothing$. Let $H_{i}=X \cap f^{-1}\left(G_{i}\right)$, $i=1, \cdots, n$. Then $H_{1}, \cdots, H_{n} \in \mathscr{H} \subset \mathscr{\mathscr { F }}$. By Lemma $9, \bigcap_{i=1}^{n} \mathrm{Cl}_{K}\left(G_{i}\right)=$ $\bigcap_{i=1}^{n} K \cap \mathrm{Cl}_{Y}\left(X \cap f^{-1}\left(G_{i}\right)\right)=\bigcap_{i=1}^{n} K \cap \mathrm{Cl}_{Y^{\prime}}\left(H_{i}\right)$ so $\bigcap_{i=1}^{n} \mathrm{Cl}_{Y}\left(H_{i}\right) \neq \varnothing$. Since $\mathscr{F}$ has the trace property with respect to $X, \bigcap_{i=1}^{n} H_{i} \neq \varnothing$. If $t \in$ $\bigcap_{i=1}^{n} H_{i}$, then $f(t) \in \bigcap_{i=1}^{n} G_{i}$. Thus $\overline{\mathscr{G}}$ has the trace property with respect to $S$.

Now suppose $\bar{G}$ has the trace property with respect to $S$. We apply Theorem 1. Let $H_{1}, \cdots, H_{n} \in \mathscr{H}$ such that $K \cap \bigcap_{i=1}^{n} \mathrm{Cl}_{Y}\left(H_{i}\right) \neq \varnothing$, and let $L$ be co-compact in $X$. There exist $G_{1}, \cdots, G_{n} \in \mathscr{G}$ such that $H_{i}=X \cap f^{-1}\left(G_{i}\right), i=1, \cdots, n$. Now,

$$
\varnothing \neq K \cap \bigcap_{i=1}^{n} \mathrm{Cl}_{Y^{(}}\left(H_{i}\right)=\bigcap_{i=1}^{n} K \cap \mathrm{Cl}_{Y^{-}}\left(X \cap f^{-1}\left(G_{i}\right)\right)=\bigcap_{i=1}^{n} \mathrm{Cl}_{K^{-}}\left(G_{i}\right) .
$$


Since $\overline{\mathscr{G}}$ has the trace property with respect to $S, \bigcap_{i=1}^{n} G_{i} \neq \varnothing$, and $\varnothing \neq \bigcap_{i=1}^{n} G_{i} \subset S=f[L]$. If $t \in L$ such that $f(t) \in \bigcap_{i=1}^{n} G_{i}$, then $t \in L \cap$ $\bigcap_{i=1}^{n} H_{i}$.

Proof of 2. By Lemma 9, $\bar{G}=\left\{K \cap C_{Y}(H) \mid H \in \mathscr{H}\right\}$. Thus, by Theorem 2 , if $\mathscr{F}$ is separating on $Y$, then $\overline{\mathscr{G}}$ is separating on $K$. We next apply Lemma 3. Let $H \in \mathscr{H}$ such that $K \subset \mathrm{Cl}_{Y}(H)$. We must show $H$ is co-compact in $X$. There exist $G \in \mathscr{G}$ such that $H=X \cap f^{-1}(G)$. Thus, $K=K \cap \mathrm{Cl}_{Y}(H)=K \cap \mathrm{Cl}_{Y}\left(X \cap f^{-1}(G)\right)=\mathrm{Cl}_{K}(G)$ and so $S=S \cap K=S \cap$ $\mathrm{Cl}_{K}(G)=\mathrm{Cl}_{S}(G)=G$ since $G$ is closed in $S$. Thus, since $f[X]=S$, $H=X \cap f^{-1}(G)=X \cap f^{-1}(S)=X$ and $H$ is co-compact in $X$. Thus, by Lemma 3 , if $\mathscr{G}$ is separating on $K$, then $\mathscr{F}$ is separating on $Y$.

Statement (3) is an immediate consequence of (1) and (2) so the proof of our theorem is complete.

All of the results of this section remain true if we merely assume that $K$ is a neighborhood retract of $Y$ instead of assuming that $K$ is a retract of all of $Y$. There are two different approaches to proving the theorems which result from this change. First, the proofs given above can be easily modified to yield the desired proofs. An alternative approach is to use results from the author's paper [2] which establish a one-to-one correspondence between compactifications of $X$ and compactifications of a co-compact subset of $X$.

IV. On the existence of retracts. The following theorem is suggested by a theorem due to A. K. Steiner and E. F. Steiner [9].

THEOREM 11. Let $X$ be locally compact and noncompact. Let $K$ be a regular Wallman space and suppose there exists a continuous map $g: X \rightarrow K$ such that whenever $L$ is co-compact in $X$, we have $g[L]$ is dense in $K$. Then there exists a compactification $Y$ of $X$ such that $K=Y-X$ and $Y$ is a Wallman compactification of $X$.

Proof. Let $X^{*}=X \cup\{\omega\}$ be the one point compactification of $X$. Let $G$ be the graph of $g$ in $X \times K$. Then, as the Steiners point out [9], the closure of $G$ in $X^{*} \times K$ is a compactification $Y$ of $X$ with the remainder $K$. Now, if we let $f: Y \rightarrow K$ be defined by $f(x, y)=y$, then $f$ is a retract map of $Y$ onto $K$. Thus, by Theorem 6 in $\S I I I$ above, $Y$ is a Wallman compactification of $X$.

We now turn our attention to a characterization of those continuous maps $g: X \rightarrow K$ which can be extended to a retract map $f: Y \rightarrow K$.

THEOREM 12. Let $g: X \rightarrow K$ be a continuous map such that $\mathrm{Cl}_{K}(H) \supset$ $K \cap \mathrm{Cl}_{Y}\left(g^{-1}(H)\right)$ for $H \subset K$. Then $g$ can be extended to a retract map of $Y$ onto $K$. 
Proof. Let $f: Y \rightarrow K$ be defined by $f(y)=g(y), y \in X$, and $f(y)=y$, $y \in K$. Let $H$ be closed in $K$. Since $f^{-1}(H)=H \cup g^{-1}(H)$ then

$$
\begin{aligned}
\mathrm{Cl}_{Y}\left(f^{-1}(H)\right) & =H \cup \mathrm{Cl}_{Y}\left(g^{-1}(H)\right)=(X \cup K) \cap\left[H \cup \mathrm{Cl}_{Y}\left(g^{-1}(H)\right)\right] \\
& =H \cup g^{-1}(H) \cup\left[K \cap \mathrm{Cl}_{Y}\left(g^{-1}(H)\right)\right] \subset f^{-1}(H) \cup \mathrm{Cl}_{K}(H)
\end{aligned}
$$

and due to the fact that $\mathrm{Cl}_{K}(H)=H \subset f^{-1}(H)$, we have $\mathrm{Cl}_{Y}\left(f^{-1}(H)\right) \subset$ $f^{-1}(H)$. Therefore, $f^{-1}(H)$ is closed and $f$ is continuous.

Combining this result with Lemma 9 , we obtain the following.

Corollary 13. Let $g: X \rightarrow K$ be a continuous map with a co-compact image $S$. Then $g$ can be extended to a retract map of $Y$ onto $K$ iff whenever $P \subset S$, we have $\mathrm{Cl}_{K}(P)=K \cap \mathrm{Cl}_{Y}\left(g^{-1}(P)\right)$.

COROLlaRY 14. Let $K$ be a regular Wallman space and let $g: X \rightarrow K$ be continuous. Then $g$ can be extended to a retract map of $Y$ onto $K$ iff whenever $P$ is regular closed in $K$, we have $P=K \cap \mathrm{Cl}_{Y}\left(g^{-1}(P)\right)$.

Proof. The hypothesis $P=K \cap \mathrm{Cl}_{Y}\left(g^{-1}(P)\right)$ for $P$ regular closed in $K$ implies $K \cap \mathrm{Cl}_{Y}\left(g^{-1}(H)\right) \subset \mathrm{Cl}_{K}(H)$ for arbitrary $H \subset K$. Thus, Lemma 5 and Theorem 12 apply.

Corollary 15. If $S$ is dense in $K$, then any retract map of $X \cup S$ onto $S$ can be extended to a retract map of $Y$ onto $K$.

Proof. Let $h: X \cup S \rightarrow S$ be a retract map. Let $g=h \mid X$. Now show that for any $H \subset K$, we have $K \cap \mathrm{Cl}_{Y}\left(g^{-1}(H)\right) \subset \mathrm{Cl}_{K}(H)$ and apply Theorem 12 .

V. Applications. It follows from Theorem 6 that if a locally compact space $X$ has a compactification $Y$ with a metric remainder $K$ such that $K$ is a retract of $Y$, then $Y$ is a Wallman compactification of $X$. For example, suppose $K$ is a metric space which is an absolute retract in the category of normal spaces. Then $K$ is a Peano space [5, pp. 18-19], [7, p. 117]. Also, suppose $X$ is any locally compact space which is not pseudocompact. Then by a theorem of Rogers [8], there exists a compactification $Y$ of $X$ with remainder $K$. Since $K$ is an absolute retract, $K$ is a retract of $Y$. Thus, by Theorem 6 above, $Y$ is a Wallman compactification of $X$.

In the example of the preceding paragraph, if we merely assume $K$ is an absolute neighborhood retract, then $K$ may not be connected. Thus, we have no guarantee that a suitable compactification $Y$ exists. Nevertheless, if we assume that the compactification $Y$ with remainder $K$ does exist, then it must be Wallman. If we know $Y$ exists, we can dispense with the non-pseudo-compactness hypothesis on $X$.

Another application of Theorem 6 is the following: Let $Y$ be a compactification of a locally compact space $X$ such that the remainder $K$ is a 
regular Wallman space. If, moreover, $K$ is an absolute retract in the category of normal spaces, then the hypotheses of Theorem 6 are satisfied and thus, $Y$ is a Wallman compactification of $X$. These considerations make the following special case of Frink's problem interesting: Must a compact absolute retract be regular Wallman? The author does not know the answer to this question.

In the preceding paragraph, we can replace "absolute retract" by "absolute neighborhcod retract".

\section{REFERENCES}

1. R. A. Alo and H. L. Shapiro, A note on compactifications and semi-normal spaces, J. Austral. Math. Soc. 8 (1968), 102-108. MR 37 \#3527.

2. H. L. Bentley, Some Wallman compactifications of locally compact spaces, Fund. Math. (to appear).

3. E. S. Berney, On Wallman compactifications, Notices Amer. Math. Soc. 17 (1970), 215. Abstract \#672-464.

4. C. M. Biles, Wallman-type compactifications (unpublished notes).

5. K. Borsuk, Theory of retracts, Monografie Mat., Tom 44, PWN, Warsaw, 1967. MR 35 \#7306.

6. O. Frink, Compactifications and semii-normal spaces, Amer. J. Math. 86 (1964), 602-607. MR $29 \# 4028$.

7. J. G. Hocking and G. S. Young, Topology, Addison-Wesley, Reading, Mass., 1961. MR 23 \#A2857.

8. J. Rogers, On compactifications with continua as remainders (to appear).

9. A. K. Steiner and E. F. Steiner, Compactifications as closures of graphs, Fund. Math. 63 (1968), 221-223. MR 38 \#6546.

10. - Products of compact metric spaces are regular Wallman, Nederi. Akad. Wetensch. Proc. Ser. A 71=Indag. Math. 30 (1968), 428-430. MR 40 \#867.

11. - Wallman and Z-compactifications, Duke Math. J. 35 (1968), 269-275. MR 37 \#3526.

12. E. F. Steiner, Wailman spaces and compactifications, Fund. Math. $61(1967 / 68)$, 295-304. MR 36 \#5899.

Department of Mathematics, University of Toledo, Toledo, Ohio 43606 